\title{
Metabolic disturbances in schizophrenia: Association with disease and drugs
}

\author{
Dr. Anant D Patil ${ }^{1}$ \\ ${ }^{I}$ (Freelance Consultant: Medical Communication and Training)
}

\begin{abstract}
Schizophrenia is a chronic and complex psychiatry disorder. Metabolic complications are commonly seen in patients with schizophrenia both in drug naive as well as treatment experienced patients. The disease itself is known for some of the derangements in metabolic parameters. Similarly, many of the antipsychotics especially second generation antipsychotics can cause metabolic disturbances which may complicate the management of schizophrenia. Long duration of treatment with second generation antipsychotics carry high risk of metabolic syndrome. It is important to select an antipsychotic with no or less potential to cause such abnormalities while treating schizophrenia. Selection of the right antipsychotic based on the potential to cause metabolic disturbances can be one of the important factors for successful outcome of the disease management. Similarly, regular screening of metabolic parameters in schizophrenia patients and treatment of these complications is equally important. Multi-disciplinary approach may be more beneficial in detecting and managing these complications.
\end{abstract}

Key words: Antipsychotic, metabolic disturbance, schizophrenia

\section{INTRODUCTION:}

Schizophrenia is a chronic, complex mental disorder. Antipsychotic medicines are the mainstay of treatment of schizophrenia ${ }^{[1]}$. Antipsychotic medication are divided into two major classes i.e. conventional antipsychotic medications referred to as either "typical" or "first-generation" antipsychotics and "atypical" or "secondgeneration" antipsychotics ${ }^{[1]}$. Patients suffering from schizophrenia are at higher risk of morbidity and mortality compared to general population. Metabolic disturbances are commonly observed during the management of schizophrenia and the drugs are often considered as causative agents for these adverse events. Some antipsychotics have more propensity of causing metabolic complications; however the disease itself may also pose risk for some of the metabolic derangements. The current article discusses the metabolic disturbances associated with schizophrenia and the antipsychotic drugs.

\section{METABOLIC DISTURBANCES ASSOCIATED WITH DISEASE:}

A study which determined twenty-five year mortality of a community cohort with schizophrenia has reported that patients with schizophrenia have a two to three times higher mortality compared to general population. The risk of increased mortality is probably life-long. This analysis also suggests that the cardiovascular mortality in schizophrenia has increased over the past 25 years compared to general population ${ }^{[2]}$.

Metabolic syndrome and cardiovascular disease risk factors are commonly seen in the patients with schizophrenia. Unhealthy lifestyle in patients with schizophrenia may one of the factors responsible for risk for the complications. Factors like poor diet and sedentary behaviour may be responsible for the risk associated with schizophrenia ${ }^{[3]}$.

Smoking also has been studied as one of the associated factors in patients with schizophrenia. As compared to general population, male patients of schizophrenia have a heavier smoking pattern ${ }^{[4]}$. The high mortality in schizophrenia can probably be attributed to the effects of cigarette smoking ${ }^{[2]}$. High rate of tobacco smoking are related to the effect of nicotine on the neurobiology of schizophrenia ${ }^{[5]}$. Improvement in negative symptoms associated with smoking may be the reason of heavy smoking in schizophrenia patients. This has been studied by Jiang $\mathbf{J}$ et al (2013) in Chinese population. In this study the investigators reported that schizophrenia patients had significantly heavier smoking pattern compared with healthy controls $(42.4 \%$ vs. $16.8 \%, \mathrm{p}<0.001$ for current smoking prevalence; $24.5 \%$ vs. $3.0 \%, \mathrm{p}<0.001$ for heavy smoker proportion). Another hypothesis for the heavier smoking suggested is that smoking may reduce side effects of antipsychotics, but in this study no significant association was seen between smoking and antipsychotics usage or risk of 
extrapyramidal side effects ${ }^{[4]}$. Smoking is associated with the induction of the CYP $1 \mathrm{~A} 2$ enzyme, and can cause reduction in the levels of drugs metabolized by CYP $1 \mathrm{~A} 2$ enzyme $^{[6]}$.

A literature review conducted by Thakore JH (2004) to find out whether schizophrenia independent of medication use is associated with the development of certain metabolic disturbances gives indications that schizophrenia is associated with an increase in visceral fat distribution and impaired fasting glucose levels independently of medication use. These abnormalities may be possibly due to hypothalamic-pituitary-adrenal (HPA) axis dysfunction. Drug-naive patients with first episode schizophrenia may have important metabolic disturbances, including central obesity and impaired fasting glucose levels ${ }^{[7]}$.

Another cross sectional study conducted to find out the prevalence of impaired fasting glucose tolerance in first-episode, drug-naive patients with schizophrenia showed more than $15 \%$ of the drug-naive patients had impaired fasting glucose tolerance, compared to none of the healthy volunteers. Similarly, the patients with schizophrenia had significantly higher fasting plasma glucose, insulin and cortisol levels and also the patients were more insulin resistant ${ }^{[8]}$.

It is well known that abdominal obesity is a significant cardiovascular risk factor ${ }^{[9]}$. However, the normal weight, drug naïve, first episode schizophrenia patients also have shown some changes in their inflammatory status. They seem to have an up-regulated inflammatory status. The results from recently published controlled study showed elevated levels of IL-1 $\beta$, IL-6, and TNF- $\alpha$ in these patients. The levels of adiponectin were significantly higher in the normal weight, drug naive patients with first episode of schizophrenia; the levels were significantly lower in the otherwise healthy obesity group ${ }^{[10]}$.

Above literature findings support the view point that metabolic disturbances can be a part of disease i.e. schizophrenia itself.

\section{DRUG INDUCED METABOLIC DISTURBANCES IN SCHIZOPHRENIA:}

Antipsychotic medicines are routinely used in the treatment of schizophrenia. Atypical or second generation antipsychotics can cause metabolic disturbances and weight gain. The frequency of these adverse events vary between different atypical antipsychotics.

A prospective interventional study from Jaipur, India has shown high prevalence of metabolic syndrome in patients treated with second generation antipsychotics. Out of 120 patients studied, $11.66 \%$ developed metabolic syndrome after 4 months of treatment. Among the 4 antipsychotics studies (haloperidol, clozapine, olanzapine and risperidone), olanzapine has shown to have maximum potential to cause metabolic syndrome. No patient out of 30, who received haloperidol developed metabolic syndrome while $23.3 \%$ patients developed metabolic syndrome with olanzapine. The incidence of metabolic syndrome with clozapine and risperidone was $13 \%$ and $10 \%$ respectively ${ }^{[11]}$.

Another recently published study in 100 subjects (50 patients of schizophrenia and 50 controls) from India has also shown higher prevalence of metabolic syndrome in patients suffering from schizophrenia (28\%) compared to general population (12\%). More than 6 month duration of treatment and use of second generation antipsychotic medicines were associated with high prevalence of metabolic syndrome. None of the patient who received first generation antipsychotics i.e. haloperidol (6 cases) or trifluperazine ( 3 cases) had metabolic syndrome whereas 9 out of 30 patients receiving olanzapine and 5 out of 5 patients receiving clozapine had metabolic syndrome ${ }^{[12]}$.

Reduced methylenetetrahydrofolate reductase (MTHFR) activity, resulting in altered metabolism of folate and hyperhomocysteinemia, may be the cause for cardiovascular disease in patients receiving atypical antipsychotics. The results from a small study have suggested the MTHFR 677C/T variant may predispose patients to atypical antipsychotic associated metabolic derangements ${ }^{[13]}$.

There is difference between second generation antipsychotics in the potential for causing weight gain. A multicenter, double-blind, randomized comparative study of amisulpride versus risperidone, in patients with chronic schizophrenia (DSM IV) with a recent worsening of symptoms in 309 patients who either received amisulpride (400-1000 mg/day) or risperidone (4-10 mg/day) for six months showed that both the treatments 
were well tolerated. During the 6-month period, significantly less patients treated with amisulpride had weight gain of $\geq 7 \%$ from baseline compared to patients who received risperidone ${ }^{[14]}$.

\section{CONCLUSION:}

Evidence shows that schizophrenia is linked with metabolic disturbances even in drug naive patients. Antipsychotics especially, second generation drugs are more likely to cause metabolic disturbances compared to first generation antipsychotics. There are differences in the propensity of causing metabolic complications among second generation antipsychotics. In high risk patients, careful selection of second generation antipsychotic is important. Regular screening may help to timely identify the early metabolic changes and avoid further complications. Collaboration between psychiatrist and endocrinologist/diabetologist is recommended for avoiding these complications and in turn improving the outcome of schizophrenia management.

\section{REFERENCES:}

[1.] Walker E, Kestler L, Bollini A, Hochman KM. Schizophrenia: Etiology and Course. Annu. Rev. Psychol. 2004. 55:401-30

[2.] Steve Brown, Miranda Kim, Clemence Mitchell and Hazel Inskip. Twenty-five year mortality of a community cohort with schizophrenia. The British Journal of Psychiatry. 2010. 196, 116-121

[3.] Hert MD, Schreurs V, Vancampfort D, Winkel RV. Metabolic syndrome in people with schizophrenia: a review. World Psychiatry 2009:8:15-22

[4.] Jiang J, See YM, Subramaniam M, Lee J. Investigation of Cigarette Smoking among Male Schizophrenia Patients. PLoS One 2013 Aug15; 8 (8):e e71343. doi: 10.1371/journal.pone.0071343.

[5.] Srinivasan TN, Thara R. Smoking in schizophrenia -- all is not biological. Schizophr Res. 2002; Jul 1; 56 (1-2): 67-74

[6.] Andrade C. Schizophrenia and smoking. J Clin Psychiatry 2012; Jun; 73 (6): e725-7

[7.] Thakore JH. Metabolic disturbance in first-episode schizophrenia. British Journal of Psychiatry.2004; 184 (suppl 47): S76-S79

[8.] Ryan MC, Collins S, Thakore JH. Impaired fasting glucose tolerance in first-episode, drug-naive patients with schizophrenia. Am J Psychiatry. 2003; Feb; 160 (2): 284-9

[9.] Protopopova D, Masopust J, Maly R, Valis M, Bazant J. The prevalence of cardiometabolic risk factors and the ten-year risk of fatal cardiovascular events in patients with schizophrenia and related psychotic disorders. Psychiatria Danubina, 2012; 24;3:307-313

[10.] Song X, Fan X, Song X, Zhang J, Zhang W, Li X, Gao J, Harrington A, Zeidonis D, Lv L. Elevated levels of adiponectin and other cytokines in drug naïve, first episode schizophrenia patients with normal weight. Schizophr Res. 2013 Aug 19; pii: S0920-9964(13)00422-2

[11.] Gautam S, Meena PS. Drug-emergent metabolic syndrome in patients with schizophrenia receiving atypical (second-generation) antipsychotics. Indian J Psychiatry. 2011; 53: 128-33

[12.] Bajaj S, Varma A, Srivastava A, Verma AK. Association of metabolic syndrome with schizophrenia. Indian J Endocr Metab 2013; 17: 890-5

[13.] Ellingrod VL, Miller DD, Taylor SF, Moline J, Holman T, Kerr J. Metabolic syndrome and insulin resistance in schizophrenia patients receiving antipsychotics genotyped for the methylenetetrahydrofolate reductase (MTHFR) 677C/T and 1298A/C variants. Schizophr Res. 2008; Jan 98; (1-3): 47-54

[14.] Sechter D, Peuskens J, Fleurot O, Rein W, Lecrubier Y and the Amisulpride Study Group Amisulpride vs. Risperidone in Chronic Schizophrenia: Results of a 6-month Double-blind Study. Neuropsychopharmacology. 2002 27:1071-1081, 2002 